\title{
The influence of MRI scan position on patients with oropharyngeal cancer undergoing radical radiotherapy
}

Scott Hanvey ${ }^{1,2^{*}}$, Mark McJury ${ }^{1,2}$, Lye Mun Tho ${ }^{4}$, Martin Glegg ${ }^{2}$, Maureen Thomson ${ }^{3}$, Derek Grose ${ }^{3}$, Allan James ${ }^{3}$, Mohammed Rizwanullah ${ }^{3}$, Claire Paterson ${ }^{3}$ and John Foster ${ }^{1,2}$

\begin{abstract}
Background: The purpose of this study was to demonstrate how magnetic resonance imaging (MRI) patient position protocols influence registration quality in patients with oropharyngeal cancer undergoing radical radiotherapy and the consequences for gross tumour volume (GTV) definition and radiotherapy planning.

Methods and materials: Twenty-two oropharyngeal patients underwent a computed tomography (CT), a diagnostic MRI $\left(M R I_{D}\right)$ and an MRI in the radiotherapy position within an immobilization mask (MRI $I_{T T}$ ). Clinicians delineated the GTV on the $C T$ viewing the $M R I_{D}$ separately $\left(G T V_{C}\right)$; on the $C T$ registered to $M R I_{D}\left(G T V_{D}\right)$ and on the $C T$ registered to $M R I_{R T}\left(G T V_{R T}\right.$ ). Planning target volumes (PTVS) were denoted similarly. Registration quality was assessed by measuring disparity between structures in the three set-ups. Volumetric modulated arc therapy (VMAT) radiotherapy planning was performed for $P T V_{C}, P T V_{D}$ and $P T V_{R T}$. To determine the dose received by the reference PTV $V_{R T}$, we optimized for PTV and PTV D wile calculating the dose to PTV ${ }_{R T}$. Statistical significance was determined using the two-tailed Mann-Whitney or two-tailed paired student t-tests.
\end{abstract}

Results: A significant improvement in registration accuracy was found between $C T$ and $M R I_{R T}$ versus the MRI measuring distances from the centre of structures (geometric mean error of $2.2 \mathrm{~mm}$ versus $6.6 \mathrm{~mm}$ ). The mean $\operatorname{GTV}_{C}\left(44.1 \mathrm{~cm}^{3}\right)$ was significantly larger than $\operatorname{GTV}_{D}\left(33.7 \mathrm{~cm}^{3}, p\right.$ value $\left.=0.027\right)$ or GTV RT $\left(30.5 \mathrm{~cm}^{3}, p\right.$ value $\left.=0.014\right)$. When optimizing the VMAT plans for PTV $C$ and investigating the mean dose to PTV RT neither the dose to $99 \%$ (58.8\%) nor 95\% of the PTV (84.7\%) were found to meet the required clinical dose constraints of 90\% and 95\% respectively. Similarly, when optimizing for PTV $D$ the mean dose to PTV $V_{R T}$ did not meet clinical dose constraints for 99\% (14.9\%) nor 95\% of the PTV (66.2\%). Only by optimizing for PTV $V_{R T}$ were all clinical dose constraints achieved.

Conclusions: When oropharyngeal patients MRI scans are performed in the radiotherapy position there are significant improvements in CT-MR image registration, target definition and PTV dose coverage.

Keywords: MRI, CT, Oropharyngeal, Radiotherapy, Image registration, Patient setup

\footnotetext{
* Correspondence: scott.hanvey@ggc.scot.nhs.uk

${ }^{1}$ Clinical Physics, University of Glasgow, Wolfson Medical School Building,

University Avenue, Glasgow G12 8QQ, Scotland, UK

${ }^{2}$ Department of Clinical Physics and Bioengineering, Beatson West of

Scotland Cancer Centre, 1053 Great Western Road, Glasgow G12 OYN,

Scotland, UK

Full list of author information is available at the end of the article
} 


\section{Background}

With intensity modulated radiotherapy and volumetric modulated arc therapy (VMAT), it is possible to deliver high doses of radiation to irregular target volumes whilst sparing normal tissue, which can result in reduced severity of radiation toxicities [1]. Increased dose delivery and dose conformity has led to a greater significance on the accurate localization of the gross tumour volume (GTV) and neighbouring structures.

In radiotherapy (RT) planning, computed tomography (CT) remains the first choice since it provides accurate dosimetric information. Imaging modalities such as magnetic resonance imaging (MRI) and positron emission tomography (PET) may present advantages compared to $\mathrm{CT}$ in terms of target definition. In particular, MRI offers improved soft tissue contrast $[2,3]$ and reduced artefacts from dental amalgam; hence, MRI is the imaging modality of choice for oropharyngeal cancers [4]. MRI improves target definition for patients with head and neck [5-7], prostate [8,9] and brain [10,11] cancer.

Challenges with MRI in clinical practice include: geometric distortion [12,13]; motion related artefacts [14] and magnetic susceptibility $[15,16]$. Patient positioning must also be considered, since differing patient set-ups between modalities can result in uncertainties in the location and magnitude of the GTV and thus the dose received by the patient [17].

Patients receiving RT to the brain can undergo an MRI in the RT position within an immobilization mask, without loss of image quality over standard imaging methods [18]. An approximate image registration uncertainty of $2 \mathrm{~mm}$ occurs when registering CT image sets in the treatment position to a diagnostic MRI for patients with brain cancer [19] and therefore many RT centres routinely register these scans for RT planning. While it could be anticipated that imaging patients with oropharyngeal cancer in the treatment position would result in improved registration and target volume definition, to our knowledge there is no published evidence to suggest that using the diagnostic MRI provides results to the contrary. In addition, no publications have analyzed the dosimetric consequences of patient positioning during MRI scan acquisition.

The purpose of this study was to compare an MRI acquired with a diagnostic patient position on a standard MRI table to an MRI acquired on a flat table with custom immobilization and to determine how this affects CT-MRI registration, GTV definition, the resulting VMAT RT plans and the magnitude of the associated geometric and dosimetric errors. The results obtained would then provide an answer to whether it was necessary to obtain a planning MRI scan for patients with oropharyngeal cancer in the RT treatment position using an immobilization mask or whether a diagnostic patient position protocol would suffice.

\section{Methods}

\section{Patient group and study overview}

Twenty two patients with oropharyngeal cancer (age 37-72), being worked up for radical RT (patients being treated with curative intent), were identified for the study, regardless of tumour or nodal stage. The study protocol was approved by Local Ethics Committee (West of Scotland Research Ethics Service, Western Infirmary, Glasgow G11 6NT, Scotland, UK) and informed written consent was obtained from all patients. Registration quality assessment was conducted on all patients, however, in three patients a GTV evaluation was not possible, since two patients had undergone primary surgical resection and another had received induction chemotherapy, resulting in complete response.

Patients underwent a CT planning scan, and two MRI scans (patients scanned between February 2010 and January 2012; median time between CT and MRI scans = 5 days, range $=0$ to 21 days). The first MRI scan was obtained with the patient in the standard diagnostic position (denoted as $M R I_{D}$ ) and the second with the patient in an immobilization mask in the RT position $\left(M_{R} I_{R T}\right)$. Both MRI scans were registered separately with the CT planning scan as shown in Figure 1. The GTV was delineated on the CT images by trained Consultant Radiation Oncologists using the treatment planning system, with the aid of viewing $\mathrm{MRI}_{\mathrm{D}}$ on a separate console. This patient position protocol is denoted as $\mathrm{PP}_{\mathrm{C}}$. This is the current standard practice at out centre, and would be considered standard practice in the majority of institutions in this country. For the purposes of this study, the GTV was delineated using two other patient set-ups. Firstly, the GTV was delineated on the $M R I_{D}$ registered to the CT datasets. This patient position protocol is denoted as $\mathrm{PP}_{\mathrm{D}}$. The GTV was also delineated on the $M R I_{R T}$ registered to the CT image sets, denoted as $\mathrm{PP}_{\mathrm{RT}}$.

\section{$\mathrm{CT}$ and MRI scanning protocol}

Patients were scanned on a GE Light-speed RT 16 slice CT scanner (GE Healthcare, WI, USA), using the current clinical scanning protocol, and immobilized within a full face and neck five point fixation thermoplastic beam directional shell (BDS) with appropriate head rest (CIVCO Medical Solutions, IA, USA). The scan extent was from superior orbital ridge to carina. A helical scan was acquired with a detector configuration of $16 \times 1.25$, pitch 0.938 , matrix $512 \times 512$ and speed $18.75 \mathrm{~mm} /$ rot with a slice thickness of $2.5 \mathrm{~mm}$.

Patients underwent MRI scans in two different positions during the same scan session. For the $\mathrm{MRI}_{\mathrm{RT}}$ scan, patients were positioned on a flat MRI Oncology Table (GE Healthcare, WI, USA), within a BDS, with a 4channel flexible surface cardiac coil positioned laterally 


\section{(a)}

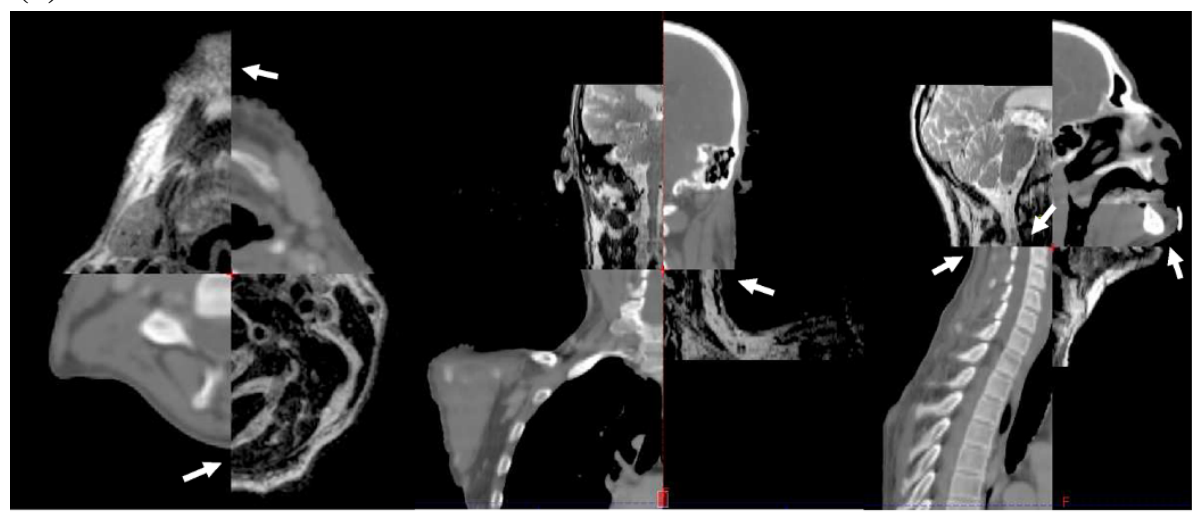

(b)

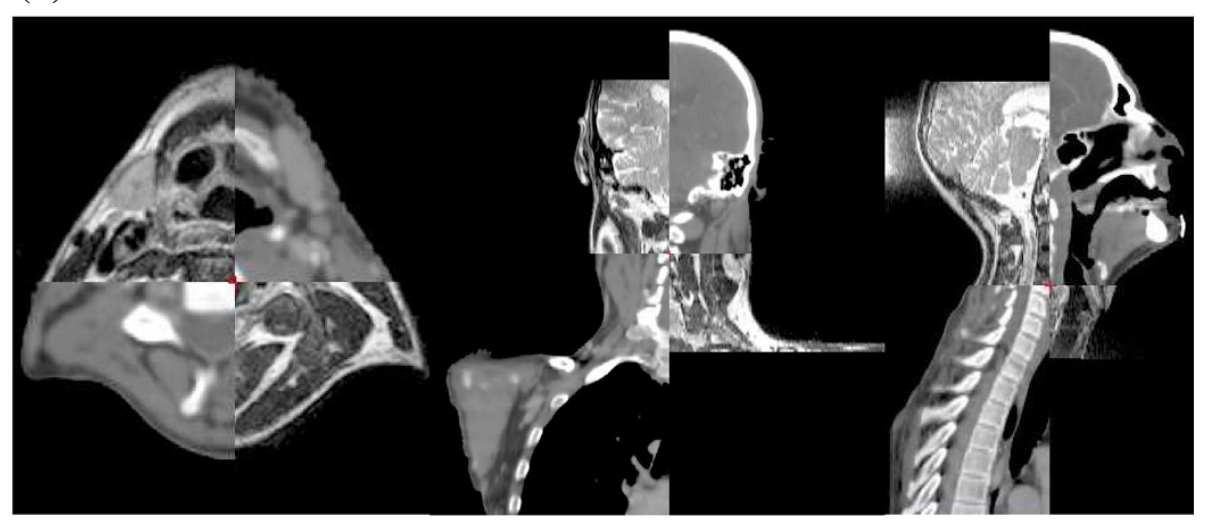

Figure 1 Split view showing the registration in the axial, coronal and sagittal planes of (a) the CT and MRI $\mathrm{I}_{\mathrm{D}}$ and (b) the CT and MRI datasets. Arrows indicate regions where there is a registration mismatch between $C T$ and $M R I_{D}$. Typically, more discrepancies in registration occurred with $M R I_{D}$ than $M R I_{R T}$. MRID : diagnostic MRI scan; MRI RT: radiotherapy positioned MRI scan.

over the patient's neck (Figure 2). The same scan extent as the CT scan was employed and the imaging parameters, chosen in accordance with local protocol, are presented in Table 1.

The scan was repeated for the $\mathrm{MRI}_{\mathrm{D}}$ on a standard curved diagnostic table without BDS using a 16-channel head, neck and spine coil, with the CT scan extent. The 16-channel head, neck and spine coil was not used for the $\mathrm{MRI}_{\mathrm{RT}}$ scan since it is not compatible with the BDS. A previous investigation using test objects has shown the image quality obtained using the 4-channel cardiac coil is of diagnostic quality [18].

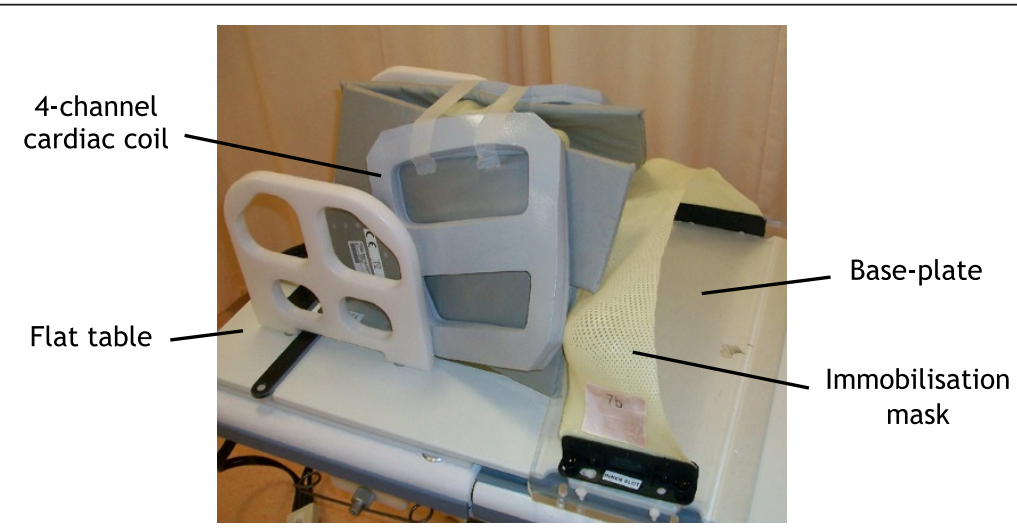

Figure 2 MRI acquired in the radiotherapy position with a 4-channel flexible surface coil positioned laterally. 
Table 1 MRI imaging parameters

\begin{tabular}{|c|c|c|c|c|c|c|c|}
\hline Scan type & FOV* $^{*}(\mathrm{~mm})$ & Slice thick (mm) & Spacing (mm) & TE† (ms) & TRtt (ms) & $\begin{array}{l}\text { Bandwidth } \\
( \pm \mathrm{kHz})\end{array}$ & Matrix size \\
\hline 2D Driven-equilibrium FSE (T2-weighted) & 400 & 2.5 & 0 & 94 & 2620 & 63 & $512 \times 256$ \\
\hline $\begin{array}{l}\text { 3D Spoiled gradient echo (T1-weighted } \\
\text { post-contrast) }\end{array}$ & 400 & 2.5 & 0 & 2 & 15 & 50 & $256 \times 256$ \\
\hline
\end{tabular}

\section{Image registration}

The treatment planning system (Eclipse, Varian Medical Systems, Inc. CA, USA, version 10.0.39) was used to register the $M R I_{R T}$ and $M R I_{D} T 1$ and T2-weighted scans to the CT data. This software enabled fully automated, mutual-information based rigid registration to be performed. While deformable registration is available for Eclipse, it does not allow inter-modality registration. Since commercial inter-modality deformable image registration is generally not available, nor employed clinically in our centre at present, this was not subject to further investigation. The volume over which the registration was performed was centred over the oropharyngeal region and chosen to include as much information for the registration algorithm as possible [6]. Fully automated registration was used without manual adjustment since, for this site, satisfactory registration for both target and nodes is not always achievable and would introduce subjective errors.

To quantitatively assess the quality of registration the orbits and the odontoid process were outlined on the CT and T1-weighted MR image sets. These three structures were delineated on the $\mathrm{CT}$, for each patient, and then repeated on the MR datasets registered to the CT.

Two metrics determined the quality of registration. Firstly, the distance between the centre of the orbits and odontoid process drawn on the CT and the MRI datasets was calculated using the coordinate location of the centre of the structures determined by the treatment planning software. The geometric mean was calculated to ensure normality of the data. Secondly, the quality of registration was assessed by measuring the spatial overlap of these structures drawn on CT and MRI. The spatial overlap was assessed by calculating the Dice coefficient (spatial overlap) for each structure, which is given by,

$$
\text { spatial overlap }=\frac{\mathrm{CT} \cap \mathrm{MRI}}{(\mathrm{CT}+\mathrm{MRI}) / 2}
$$

where CT $\cap$ MRI is the volume of intersection between the CT and MRI structures. The value of the spatial overlap can range from zero, which indicates no spatial overlap between the CT and MRI volumes, to one, which indicates complete overlap [20]. Since structures outlined on CT and MRI may differ, even with perfect registration, a spatial overlap of one may not be achieved in practice, but will still be dependent on different patient set-up and registration quality.

\section{Gross tumour volume, lower risk clinical target volume and organ at risk delineation}

Three Oncologists were assigned five patients each and a fourth was assigned four patients. The Oncologists delineated the GTV on their patients using the three set-ups $\mathrm{PP}_{\mathrm{C}}, \mathrm{PP}_{\mathrm{D}}$ and $\mathrm{PP}_{\mathrm{RT}}$, which in this study are referred to as $\mathrm{GTV}_{\mathrm{C}}, \mathrm{GTV}_{\mathrm{D}}$ and $\mathrm{GTV}_{\mathrm{RT}}$ respectively. Anonymized information sheets, containing the patient's clinical history and radiology report, were available to the Oncologists. The clinicians generally utilized the T2-weighted MR datasets while contouring the GTV, although T1-weighted images were also referenced. A period of at least a week was given between delineations of the GTV for the same patient using a different imaging protocol and the Oncologists were blinded to previous delineations. Changes in the magnitude of the GTV were assessed. Contouring was also performed on the lower risk clinical target volume (CTV LR) of the nodal areas at risk of microscopic involvement for a randomly selected cohort of ten patients, in each of the set-ups. Nodal delineation was performed according to international consensus guidelines [21]. For these patients, the organs at risk (OARs), which included the left and right parotids, larynx, spinal cord and the brainstem, were also contoured.

The GTV and CTV LR were expanded to obtain planning target volumes, (PTV) and lower risk planning target volumes (PTV LR) respectively. To create the clinical target volume (CTV) the GTV was expanded by $1 \mathrm{~cm}$ isotropically, removing any overlap with bone or air cavities. The CTV was then enlarged by $3 \mathrm{~mm}$ isotropically and cropped from the external outline of the body to create the PTV. It is necessary to crop the PTV from the body outline to assist in the VMAT optimization process. The PTVs were generated from the GTVs using the three setups $\mathrm{PP}_{\mathrm{C}}, \mathrm{PP}_{\mathrm{D}}$ and $\mathrm{PP}_{\mathrm{RT}}$, which are denoted as $\mathrm{PTV}_{\mathrm{C}}$, $\mathrm{PTV}_{\mathrm{D}}$ and PTV $\mathrm{RT}_{\mathrm{R}}$ respectively. Similarly, to generate the PTV LR the CTV LR was expanded by $3 \mathrm{~mm}$ isotropically and cropped by an appropriate margin from the body outline. Target volumes and OARs were generated in accordance with local protocol. 


\section{Dose analysis}

VMAT plans were calculated for the ten patients for whom the GTV, CTV LR and OARs were contoured, to determine the impact that changes in target volume definition have on RT planning. The VMAT plans were calculated in accordance with our centre's clinical dose constraints using the Anisotropic Analytical Algorithm and Progressive Resolution Optimizer VMAT algorithm in Eclipse (Varian Medical Systems, Inc, CA, USA) version 10.0.28. A VMAT plan was calculated for $\mathrm{PTV}_{\mathrm{C}}$, $\mathrm{PTV}_{\mathrm{D}}$ and $\mathrm{PTV}_{\mathrm{RT}}$. Dose volume histograms (DVHs) were generated for $\mathrm{PTV}_{\mathrm{C}}, \mathrm{PTV}_{\mathrm{D}}$ and $\mathrm{PTV}_{\mathrm{RT}}$. A mean DVH for all ten patients was then calculated for $\mathrm{PP}_{\mathrm{C}}, \mathrm{PP}_{\mathrm{D}}$ and $\mathrm{PP}_{\mathrm{RT}}$. These results are presented using our centre's dose constraint protocol i.e. $\mathrm{D}_{99}>90 \%, \mathrm{D}_{95}>95 \%$, $\mathrm{D}_{5}<105 \%$ and $\mathrm{D}_{2}<107 \%$, where $\mathrm{D}_{99}>90 \%$ means $99 \%$ of the total PTV volume should receive a dose $>90 \%$ of the prescribed dose. The other dose constraints are defined similarly.

Of particular interest in this study was to establish the quality of the RT plan for $\mathrm{PP}_{\mathrm{C}}$ and $\mathrm{PP}_{\mathrm{D}}$ with reference to $\mathrm{PP}_{\mathrm{RT}}$, since our working hypothesis postulates that the optimum target volume definition is achieved with $\mathrm{PP}_{\mathrm{RT}}$. To achieve this we optimized for $\mathrm{PTV}_{\mathrm{C}}$ and $\mathrm{PTV}_{\mathrm{D}}$ but investigated the dose coverage of $\mathrm{PTV}_{\mathrm{RT}}$ at $\mathrm{D}_{99}>90 \%$, $\mathrm{D}_{95}>95 \%, \mathrm{D}_{5}<105 \%$ and $\mathrm{D}_{2}<107 \%$.

A quantitative comparison of the homogeneity of the dose to the PTV was completed using the sigma index. The sigma index was compared individually for $\mathrm{PTV}_{\mathrm{C}}$, $\mathrm{PTV}_{\mathrm{D}}$ and PTV $\mathrm{RT}_{\mathrm{T}}$ as well as for PTV $\mathrm{C}, \mathrm{PTV}_{\mathrm{D}}$ with reference to $P T V_{R T}$. The sigma index is equal to the standard deviation of the dose throughout the PTV, calculated on a voxel by voxel basis [22], thus the higher the sigma index, the greater the dose inhomogeneity.
Finally, the dose to the OARs was assessed by evaluating the mean and maximum dose received using $\mathrm{PP}_{\mathrm{C}}$, $\mathrm{PP}_{\mathrm{D}}$ and $\mathrm{PP}_{\mathrm{RT}}$.

Two-tailed paired student t-tests were performed to examine the statistical differences of the registration quality and dosimetric indices, except for the geometric mean distance from the centre of structures in CT and MRI where two-tailed Mann-Whitney tests were performed. A Mann-Whitney test was used to assess the geometric mean distance from the centre of the structures because the data was not normally distributed. The null hypothesis was rejected when the $p$ value was less than 0.05 .

\section{Results}

There was a reduction in the geometric mean distance from the centre of the orbits and odontoid process delineated on the CT and $\mathrm{MRI}_{\mathrm{RT}}$ to that delineated on the CT to $M R I_{D}$ volumes (Figure 3) and this was significant ( $p$ value $<0.001)$ for each structure. Narrower error bars exist for the CT registered to the $M R I_{R T}$ versus the $M R I_{D}$, which strongly implies that the patients are positioned more closely to their CT set-up in the BDS than when using the ordinary diagnostic set-up. No correlation was found between the time from the CT and MRI scan in days and the mean registration error for either $\operatorname{MRI}_{\mathrm{D}}\left(\mathrm{R}^{2}=0.06\right)$ or $\mathrm{MRI}_{\mathrm{RT}}\left(\mathrm{R}^{2}=0.02\right)$.

An improvement in the mean spatial overlap for the orbits and odontoid process was observed between $\mathrm{CT}$ and the CT-MRI $I_{R T}$ over the CT to CT-MRI $I_{D}$ data sets as shown in Table 2. Analyzing the difference between the spatial overlap of CT to CT-MRI $I_{D}$ versus $C T$ to $C T$ $\mathrm{MRI}_{\mathrm{RT}}$ was shown to have a $p$ value $<0.001$ for both

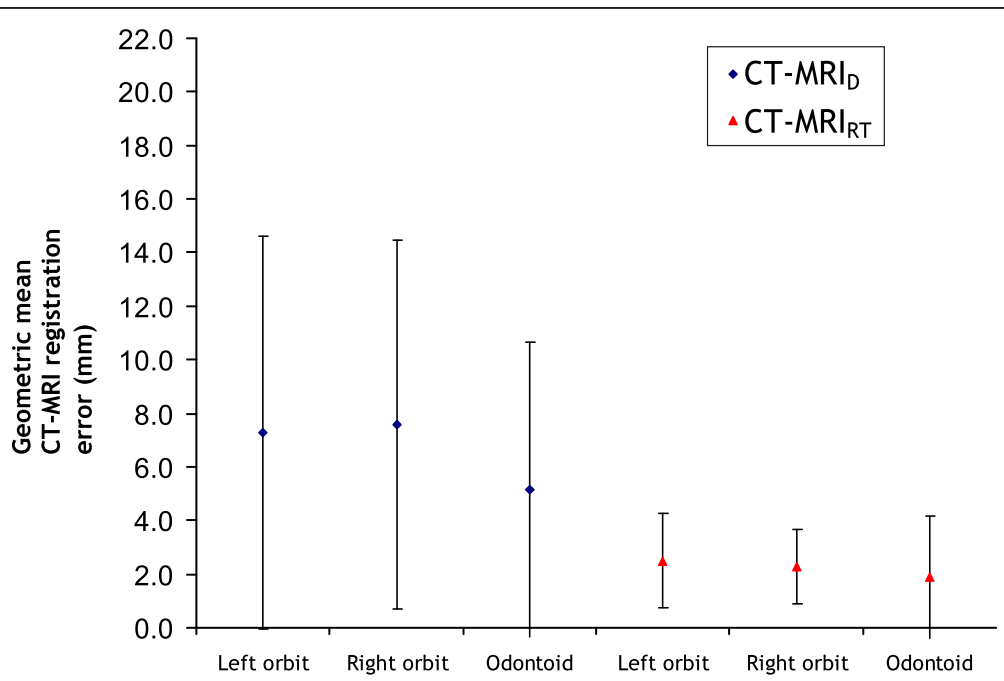

Figure 3 Quality of registration results. The geometric mean CT-MRI registration error is the geometric mean of the distance from the centre of the CT structures to the centre of the MRI structures. The error bars represent \pm 1 standard deviation. Odontoid: odontoid process; CT-MRID: $\mathrm{CT}$ registered with the diagnostic MRI scan; $C T-M_{R} I_{R T}: C T$ registered with radiotherapy positioned MRI scan. 
Table 2 Mean spatial overlap of the anatomical landmarks for the two registration set-ups

\begin{tabular}{llll}
\hline & $\begin{array}{l}\text { CT-MRI }_{\mathrm{D}}{ }^{*} \text { mean } \\
\text { spatial overlap }\end{array}$ & $\begin{array}{l}\text { CT-MRI } \\
\text { spatial overlap }\end{array}$ & $\boldsymbol{p}$ value \\
\hline Left orbit & 0.49 & 0.81 & $<0.001$ \\
Right orbit & 0.48 & 0.81 & $<0.001$ \\
Odontoid process & 0.37 & 0.67 & $<0.001$ \\
\hline
\end{tabular}

* CT registered with diagnostic MRI.

$+\mathrm{CT}$ registered with the radiotherapy positioned MRI.

orbits and odontoid process (Table 2). The volume of the three structures delineated on CT was larger than those delineated on MRI, however, their mean difference was within one standard deviation. Therefore, the spatial overlap is expected to give a good measure of the quality of registration.

The mean $\mathrm{GTV}_{\mathrm{C}}$ was significantly larger than the $\mathrm{GTV}_{\mathrm{D}}$ or $\mathrm{GTV}_{\mathrm{RT}}$ as shown in Table 3. The $p$ values in Table 3 refer to the differences in the magnitude of the $\mathrm{GTV}_{\mathrm{C}}$ and the other two GTVs. There was no significant difference between $\mathrm{GTV}_{\mathrm{D}}$ and $\mathrm{GTV}_{\mathrm{RT}}$ ( $p$ value $=$ $0.14)$.

Clinical dose constraints were met for the mean DVHs of $\mathrm{PTV}_{\mathrm{C}}, \mathrm{PTV}_{\mathrm{D}}$ and $\mathrm{PTV}_{\mathrm{RT}}$ (columns 3-5 of Figure 4 (a)), which validates the planning methodology in this study. When optimizing the RT plan for $\mathrm{PTV}_{\mathrm{C}}$, or repeating the optimization process for $\mathrm{PTV}_{\mathrm{D}}$, the dose to PTV $_{R T}$ shows that neither the $\mathrm{D}_{99}$ nor the $\mathrm{D}_{95}$ dose constraints are met for the mean DVHs of $\mathrm{PP}_{\mathrm{C}}$ or $\mathrm{PP}_{\mathrm{D}}$ (the last 2 columns of Figure 4(a) and (b)). For example, for $\mathrm{D}_{99}$ mean dose to the PTV for $\mathrm{PTV}_{\mathrm{D}}$ : $\mathrm{PTV}_{\mathrm{RT}}$ is $14.9 \%$ rather than the $90 \%$ required. Conformity to dose constraints is poorer for $\mathrm{PTV}_{\mathrm{D}}$ : $\mathrm{PTV}_{\mathrm{RT}}$ compared to $\mathrm{PTV}_{\mathrm{C}}$ : $\mathrm{PTV}_{\mathrm{RT}}$ (Figure 4(b)). Only by using $\mathrm{PP}_{\mathrm{RT}}$ can all dose constraints be achieved for PTV $\mathrm{RT}_{\mathrm{RT}}$.

The justification for choosing only ten patients to perform the dose analysis was that none of the ten patients met the $90 \%$ dose constraint for $\mathrm{PTV}_{\mathrm{C}}: \mathrm{PTV}_{\mathrm{RT}}$ and PTV $_{\mathrm{D}}$ : PTV $\mathrm{RT}_{\mathrm{RT}}$ and only one of the ten patients met the 95\% dose constraint for both $\mathrm{PTV}_{\mathrm{C}}$ : $\mathrm{PTV}_{\mathrm{RT}}$ and $\mathrm{PTV}_{\mathrm{D}}$ : $\mathrm{PTV}_{\mathrm{RT}}$. This relates to a $95 \%$ confidence interval of $0.0 \%$ to $30.9 \%$ and $0.3 \%$ to $44.5 \%$ respectively. These confidence intervals demonstrate that no further cases are statistically necessary in the analysis.

Table 3 Mean GTV* $\left(\mathrm{cm}^{3}\right)$ delineated with the different patient position protocols

\begin{tabular}{|c|c|c|}
\hline$\overline{\text { Mean GTV }}{ }_{\mathrm{C}} \dagger$ & Mean GTV ${ }_{D}+\dagger$ ( $p$ value) & Mean GTV $\mathrm{RT}_{\mathrm{RT}}^{\#}(p$ value) \\
\hline 44.1 & $33.7(0.027)$ & $30.5(0.014)$ \\
\hline
\end{tabular}

The $p$ value relates to differences in the magnitude of $\mathrm{GTV}_{\mathrm{C}}$ and the other two GTVs.

* Gross tumour volume.

† GTV delineated on CT with diagnostic MRI scan viewed separately.

†† GTV delineated using the CT registered with diagnostic MRI.

\# GTV delineated using the CT registered with radiotherapy positioned MRI.
Figure 5 demonstrates for a typical patient the PTV $\mathrm{RT}_{\mathrm{RT}}$ and the dose distributions optimized for $\mathrm{PTV}_{\mathrm{C}}$ (left) and $\mathrm{PTV}_{\mathrm{D}}$ (right). This figure shows that the 95\% isodose line does not cover $\mathrm{PTV}_{\mathrm{RT}}$ entirely with a posterior proportion of the PTV receiving a dose less than $95 \%$ of the prescribed dose. Figure 5 is in agreement with the results of Figure 4 which shows that when optimizing for $\mathrm{PTV}_{\mathrm{C}}$ or $\mathrm{PTV}_{\mathrm{D}}$ not all the dose constraints are met for $\mathrm{PTV}_{\mathrm{RT}}$.

The mean sigma indices are shown in Table 4. In the first three columns of Table 4 the sigma indices are within $3.3 \%$ again validating the planning methodology in this study. The dose homogeneity becomes considerably poorer for $\mathrm{PTV}_{\mathrm{C}}$ : $\mathrm{PTV}_{\mathrm{RT}}$ and $\mathrm{PTV}_{\mathrm{D}}$ : $\mathrm{PTV}_{\mathrm{RT}}$ at $7.3 \%$ and 9.1\% respectively. A statistically significant difference between the sigma indices was found between $\mathrm{PTV}_{\mathrm{C}} \mathrm{PTV}_{\mathrm{C}}$ and $\mathrm{PTV}_{\mathrm{C}}: \mathrm{PTV}_{\mathrm{RT}}(p$ value $=0.004)$ and between $\mathrm{PTV}_{\mathrm{D}}$ : $\mathrm{PTV}_{\mathrm{D}}$ and $\mathrm{PTV}_{\mathrm{D}}$ : $\mathrm{PTV}_{\mathrm{RT}}(p$ value $=0.008)$. The mean sigma index for $\mathrm{PTV}_{\mathrm{D}}$ : $\mathrm{PTV}_{\mathrm{RT}}$ is poorer than for $\mathrm{PTV}_{\mathrm{C}}$ : $\mathrm{PTV}_{\mathrm{RT}}$ in agreement with the results of Figure 4(a).

Results for parotid and larynx OAR dose analyses are presented in Table 5. In situations where there was overlap between a parotid and PTV, it was considered that dose sparing to that parotid was not possible without compromising PTV dose; hence it was excluded from the analysis. The results demonstrate that in planning $\mathrm{PTV}_{\mathrm{C}}, \mathrm{PTV}_{\mathrm{D}}$ and $\mathrm{PTV}_{\mathrm{RT}}$, in all 10 patients the mean dose to the parotid was $<2400$ cGy which met clinical dose constraints. However, when RT plans were optimized for $\mathrm{PTV}_{\mathrm{C}}$ and $\mathrm{PTV}_{\mathrm{D}}$ and the dose to the parotid for $\mathrm{PP}_{\mathrm{RT}}$ were analysed $\left(\mathrm{PTV}_{\mathrm{C}}\right.$ : $\mathrm{PTV}_{\mathrm{RT}}$ and $\left.\mathrm{PTV}_{\mathrm{D}}: \mathrm{PTV}_{\mathrm{RT}}\right)$ in 5 out of 10 patients the parotid dose exceeded the tolerance. For the larynx, the tolerance of 4000 cGy was exceeded in 4 out of 10 for $\mathrm{PP}_{\mathrm{C}}, 3$ out of 10 for $\mathrm{PP}_{\mathrm{D}}$ and 1 out of 10 patients for $\mathrm{PP}_{\mathrm{RT}}$. However, as with the parotid, when RT plans were optimized for $\mathrm{PTV}_{\mathrm{C}}$ and $\mathrm{PTV}_{\mathrm{D}}$ and the dose to the larynx for $\mathrm{PP}_{\mathrm{RT}}$ were analysed there were more instances of unmet dose constraints (5 out of $10 \mathrm{pa}-$ tients for both PTV $\mathrm{C}_{\mathrm{C}}$ PTV $\mathrm{RT}_{\mathrm{RT}}$ and $\mathrm{PTV}_{\mathrm{D}}$ : $\mathrm{PTV}_{\mathrm{RT}}$ ).

When the mean dose to the parotid and larynx for all 10 patients was calculated, an incrementally smaller value was seen for PTV $\mathrm{P}_{\mathrm{C}}, \mathrm{PTV}_{\mathrm{D}}$ and PTV $\mathrm{RT}$ (first 3 columns of Table 5), which can be explained by the decrease in the magnitude of the GTV. Dose to the spinal cord and brainstem were also calculated but were not found to exceed clinically relevant tolerances for any of the patient position protocols.

\section{Discussion}

Advanced imaging techniques have been shown to improve tumour and nodal staging [23] and the benefits of integrating MRI are well known [5-7,23,24]. While the advantages in positioning patients in a similar way to their CT planning scan when acquiring MR images are 
(a)

\begin{tabular}{lllllll}
$\begin{array}{l}\text { Ratio of total } \\
\text { PTV volume } \\
(\%)\end{array}$ & $\begin{array}{l}\text { Dose } \\
\text { constraint } \\
(\%)\end{array}$ & PTV $_{\mathrm{C}}: \mathrm{PTV}_{\mathrm{C}}$ & $\mathrm{PTV}_{\mathrm{D}}: \mathrm{PTV}_{\mathrm{D}}$ & $\mathrm{PTV}_{\mathrm{RT}}: \mathrm{PTV}_{\mathrm{RT}}$ & $\mathrm{PTV}_{\mathrm{C}}: \mathrm{PTV}_{\mathrm{RT}}$ & $\mathrm{PTV}_{\mathrm{D}}: \mathrm{PTV}_{\mathrm{RT}}$ \\
\hline $99 \%$ & $>90$ & 90.8 & 91.4 & 90.9 & 58.8 & 14.9 \\
$95 \%$ & $>95$ & 95.3 & 95.7 & 95.3 & 84.7 & 66.2 \\
$5 \%$ & $<105$ & 104 & 104.4 & 104 & 103.8 & 104.1 \\
$2 \%$ & $<107$ & 105.7 & 105.4 & 105.1 & 104.7 & 105.1 \\
\hline
\end{tabular}

(b)

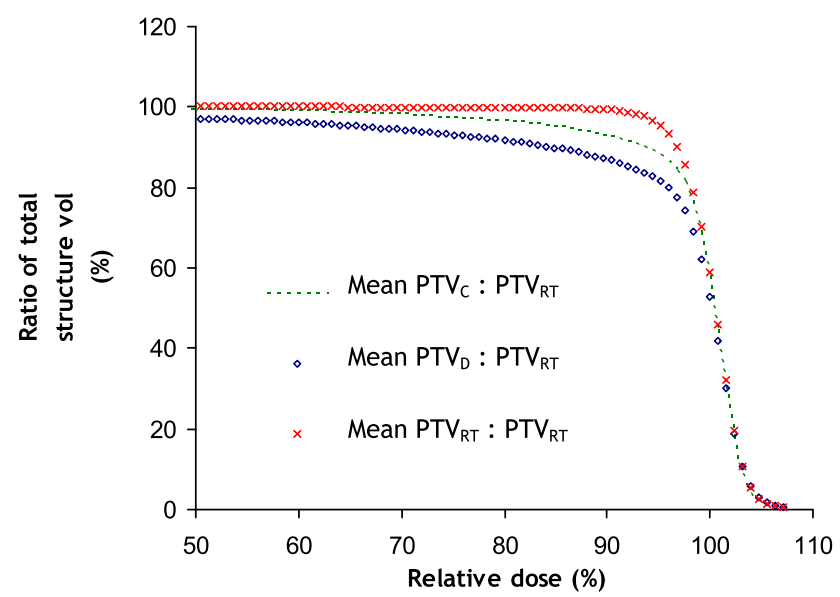

Figure 4 Mean DVH values and mean DVHs. (a) Values obtained from mean DVHs optimizing for each PTV but investigating the dose to each PTV and (b) mean DVHs optimizing for each PTV but investigating the dose to PTV RT. The PTV left of the colon indicates the PTV for which

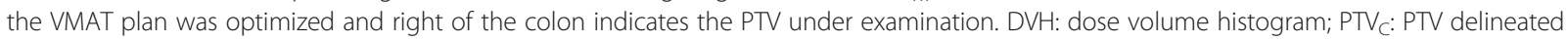
using the $C T$ with the diagnostic MRI scan viewed on a separate console; PTV : PTV delineated on the $C T$ registered with the diagnostic MRI scan; PTVRT: PTV delineated on the CT registered with the MRI scan in the radiotherapy position.

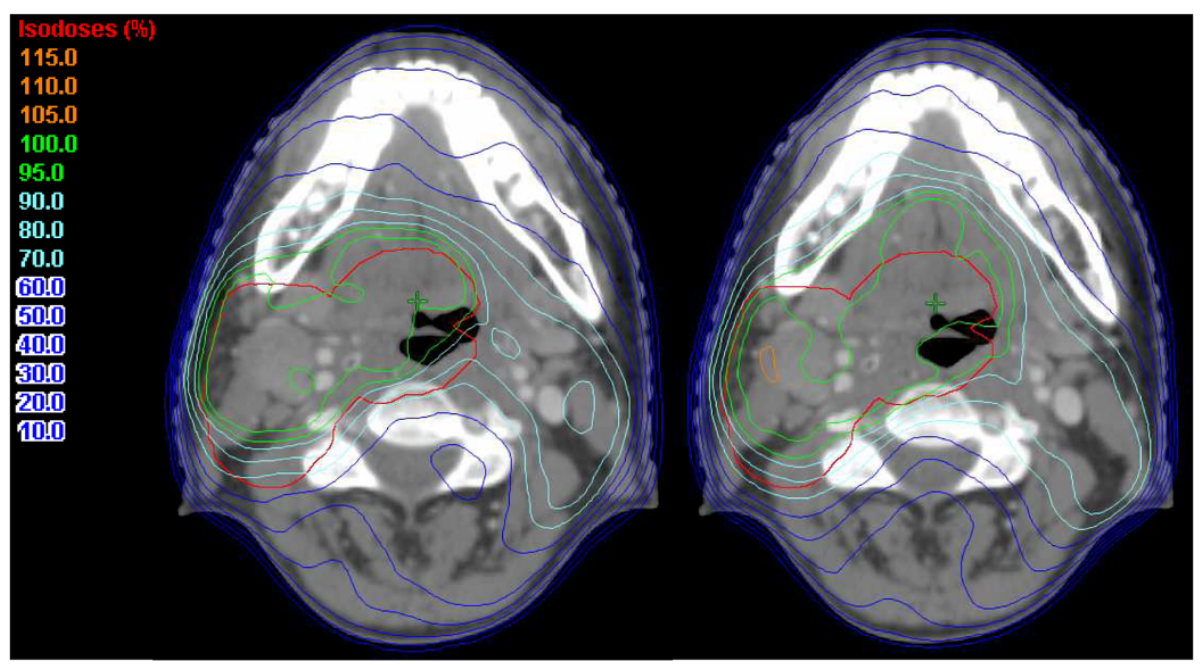

Figure 5 PTV $_{R T}$ and the dose distributions optimized for PTV $_{C}$ (left) and optimized for PTV (right). PTV $_{\text {RT: }}$ PTV delineated on the CT registered with the MRI scan in the radiotherapy position; PTV : PTV delineated using the $C T$ with the diagnostic MRI scan viewed on a separate console; PTV : PTV delineated on the CT registered with the diagnostic MRI scan. 
Table 4 Mean sigma indices for the PTVs

\begin{tabular}{|c|c|c|c|c|c|}
\hline & PTV $_{\mathrm{C}}:$ PTV $_{\mathrm{C}}^{*}$ & PTV $_{D}$ PTV $_{D} \dagger$ & PTV $_{\mathrm{RT}}:$ PTV $\mathrm{RT}_{\mathrm{RT}}+\dagger$ & PTV $_{\mathrm{C}}:$ PTV $_{\mathrm{RT}}$ & PTV $_{\mathrm{D}}: \mathrm{PTV}_{\mathrm{RT}}$ \\
\hline Mean sigma index (\%) & 3.0 & 3.1 & 3.3 & 7.3 & 9.1 \\
\hline
\end{tabular}

PTV left of the colon indicates the PTV for which the VMAT plan was optimized and right of the colon the PTV under examination.

* PTV delineated using the CT with diagnostic MRI viewed separately.

† PTV delineated on the CT registered with diagnostic MRI.

†† PTV delineated on the $C T$ registered with radiotherapy positioned MRI.

also known $[6,17,23]$, this is the first study to compare the CT-MRI registration accuracy and dosimetric effects of a diagnostic versus RT positioned MRI scan in patients with oropharyngeal cancer.

During the process of registering CT to MR images, there was improved registration with fewer discrepancies using $\mathrm{MRI}_{\mathrm{RT}}$ compared to $M \mathrm{MI}_{\mathrm{D}}$, as demonstrated in Figure 1. In this example, there are registration discrepancies at the body outline and spinal cord for CT-MRI as indicated by the arrows (Figure 1(a)), but not with $\mathrm{MRI}_{\mathrm{RT}}$ (Figure 1(b)).

A significant improvement in the registration quality of CT to $M R I_{R T}$ versus the $M R I_{D}$ was demonstrated by a reduction in the geometric mean distance from the centre of the orbits and odontoid process delineated on $\mathrm{CT}$ and the $\mathrm{MRI}_{\mathrm{RT}}$ and an increase in the spatial overlap of these structures. These results show that patient setup significantly influences CT-MRI registration accuracy. With increased interest in the use of dose escalation and dose painting techniques within RT planning the importance of improved image registration becomes ever more relevant.

The significant difference between the magnitude of the $\mathrm{GTV}_{\mathrm{C}}$ and $\mathrm{GTV}_{\mathrm{D}}$ and between the $\mathrm{GTV}_{\mathrm{C}}$ and $G_{T V}$ implies that the GTV is significantly smaller when using registered rather than unregistered CT-MR images. This underlines the importance of registering CT to MRI for patients with oropharyngeal cancer, rather than viewing them separately. It also highlights the difficulty in delineating oropharyngeal cancers with CT due to the similarity in Hounsfield Units of tumour and surrounding tissue as well as artefacts caused by dental amalgam. While there was no significant difference between the mean $G T V_{D}$ and $G T V_{R T}$, there were important differences in the VMAT plans, as discussed below. To achieve the clinical goal of reduced late toxicities and improved tumour control using dose escalation with tighter PTV margins, uncertainties in GTV delineation need to be minimized and our data suggest $\mathrm{PP}_{\mathrm{RT}}$ offers the optimal of the three set-ups.

The results reveal that there are potentially clinically relevant improvements to the quality of the VMAT plans when using $\mathrm{PP}_{\mathrm{RT}}$ rather than $\mathrm{PP}_{\mathrm{C}}$ or $\mathrm{PP}_{\mathrm{D}}$. This is demonstrated by the PTV dose coverage, PTV dose homogeneity and instances of unmet dose constraints by the OARs. Due to improved registration accuracy and MRI being the recommended imaging modality for soft tissue oropharyngeal cancers [4], it may be assumed that PTV $_{R T}$ would be the reference PTV. To determine the dose received by the reference, $\mathrm{PTV}_{\mathrm{RT}}$, we optimized for $\mathrm{PTV}_{\mathrm{C}}$ and $\mathrm{PTV}_{\mathrm{D}}$ while calculating the dose to $\mathrm{PTV}_{\mathrm{RT}}$. When optimizing for PTV $\mathrm{RT}_{\mathrm{RT}}$ it was shown that the mean DVH for $\mathrm{PTV}_{\mathrm{D}}$ had poorer dose coverage than $\mathrm{PTV}_{\mathrm{C}}$ (Figure 4). Despite the magnitude of the mean $\mathrm{GTV}_{\mathrm{RT}}$ and $G T V_{D}$ being similar there may be differences in the shape and location of the GTV using these patient set-

Table 5 Mean dose for the (a) parotid and (b) larynx

(a)

\begin{tabular}{|c|c|c|c|c|c|}
\hline & \multicolumn{5}{|c|}{ Parotid mean dose (cGy) } \\
\hline & \multicolumn{5}{|c|}{ Mean dose constraint $2400 \mathrm{cGy}$} \\
\hline & $\mathrm{PP}_{\mathrm{C}}: \mathrm{PP}_{\mathrm{C}}{ }^{*}$ & $\mathrm{PP}_{\mathrm{D}}: \mathrm{PP}_{\mathrm{D}} \mathrm{T}^{-}$ & $\mathrm{PP}_{\mathrm{RT}}: \mathrm{PP}_{\mathrm{RT}} \mathrm{T \dagger}$ & $\mathrm{PP}_{\mathrm{C}}: \mathrm{PP}_{\mathrm{RT}}$ & $P P_{D}: P P_{R T}$ \\
\hline Mean & 2179.6 & 1965.5 & 1820.3 & 2233.8 & 1992.4 \\
\hline Number of patients which exceed the dose constraint & 0 & 0 & 0 & 5 & 5 \\
\hline
\end{tabular}

(b)

\begin{tabular}{|c|c|c|c|c|c|}
\hline & \multicolumn{5}{|c|}{ Larynx mean dose (cGy) } \\
\hline & \multicolumn{5}{|c|}{ Mean dose constraint 4000 cGy } \\
\hline & PP $P_{C}: P_{C}$ & $P P_{D}: P P_{D}$ & $\mathrm{PP}_{\mathrm{RT}}: \mathrm{PP}_{\mathrm{RT}}$ & $\mathrm{PP}_{\mathrm{C}}: \mathrm{PP}_{\mathrm{RT}}$ & $\mathrm{PP}_{\mathrm{D}}: \mathrm{PP}_{\mathrm{RT}}$ \\
\hline Mean & 3979.6 & 3892.3 & 3613.2 & 4108.8 & 4020.6 \\
\hline Number of patients which exceed the dose constraint & 4 & 3 & 1 & 5 & 5 \\
\hline
\end{tabular}

Where there was overlap between a parotid and the PTV the parotid dose was not included in the mean parotid dose. PP left of the colon indicates the patient position protocol for which the VMAT plan was optimized and right of the colon indicated the patient position protocol under examination.

* Patient position protocol: CT and diagnostic MRI scan viewed separately.

† Patient position protocol: $\mathrm{CT}$ registered with the diagnostic MRI.

†† Patient position protocol: CT registered with radiotherapy positioned MRI. 
ups which would result in the DVHs of Figure 4. Neither the $\mathrm{PP}_{\mathrm{C}}$ nor the $\mathrm{PP}_{\mathrm{D}}$ VMAT plans were able to meet the clinical dose constraints of $\mathrm{D}_{99}$ and $\mathrm{D}_{95}$ for the reference, $\mathrm{PTV}_{\mathrm{RT}}$. Furthermore, it has been argued that tumour control probability can be considerably compromised by an inhomogeneous dose to the PTV [25]. It is therefore suboptimal to use $\mathrm{PP}_{\mathrm{C}}$ or $\mathrm{PP}_{\mathrm{D}}$ rather than $\mathrm{PP}_{\mathrm{RT}}$ for $\mathrm{RT}$ planning of patients with oropharyngeal cancer.

Obtaining a further MRI in the RT position, rather than using the original diagnostic MRI, may place greater demands on increasingly stretched healthcare resources. However, this must be weighed against the potential advantages of improved image registration and, by consequence, superior target volume definition and dose coverage of the PTV, as these results have demonstrated. Our study suggests further research, particularly in correlating dosimetric investigations with clinical outcome data, would be warranted.

\section{Conclusions}

When MRI scans are performed in the RT position, as opposed to using diagnostic MR images not obtained in RT position, there are significant improvements in the quality of CT-MR registration. This study has also shown that RT positioned MRI scans offer improvements in target definition, dose coverage and dose homogeneity, which could have significant implications for tumour control rates. To our knowledge, this is the first study in the literature to confirm these advantages.

\section{Abbreviations \\ BDS: Beam directional shell; CT: Computed tomography; CTV: Clinical target volume; CTV LR: Lower risk clinical target volume; DVH: Dose volume histograms; GTV: Gross tumour volume; GTVC: Gross tumour volume delineated using PP $;$; GTV : Gross tumour volume delineated using PP $P_{D}$; $\mathrm{GTV}_{\mathrm{RT}}$ : Gross tumour volume delineated using PP $\mathrm{RT}_{\mathrm{T}}$ M MRI: Magnetic resonance imaging; $M R I_{D}$ : MRI with the patient in the standard diagnostic position; $\mathrm{MRI}_{\mathrm{RT}}$ : MRI with the patient in the radiotherapy position in an immobilization mask; OAR: Organ at risk; PET: Positron emission tomography; PP : Patient position protocol with the $C T$ and $M R I_{D}$ on a separate console; $P P_{D}$ : Patient position protocol with the $C T$ and $M M_{D}$ registered; $P_{R T}$ : Patient position protocol with the $C T$ and $M R I_{R T}$ registered; PTV: Planning target volume; PTV: LR Lower risk planning target volume; PTV : Planning target volume generated from GTV $V_{C}$ PTV $V_{D}$ : Gross tumour volume generated from GTV PTV $V_{\text {RT: }}$ Gross tumour volume generated from GTVRT; RT: Radiotherapy; VMAT: Volumetric modulated arc therapy.}

\section{Competing interests}

The authors declare they have no competing interests.

\section{Authors' contributions}

SH and JF conceived the study and participated in its design. SH and MT coordinated the study. SH generated the radiotherapy plans and analyzed the data, SH, MMc, JF and MG helped draft the manuscript, LMT delineated the volumes for the radiotherapy plans and the GTVs. DG, AJ, MR and CP delineated the GTVs. All authors read and approved the final manuscript.

\section{Acknowledgements}

The authors would like to express their gratitude to the Beatson radiotherapy and diagnostic radiography staff and to Mr James Paul, Head of Biostatistics at the Clinical Trials Unit, Beatson West of Scotland Cancer Centre. We would also like to acknowledge financial support from the NHS Learning and Education and The Beatson Oncology Centre Fund.

\section{Author details}

${ }^{1}$ Clinical Physics, University of Glasgow, Wolfson Medical School Building, University Avenue, Glasgow G12 8QQ, Scotland, UK. ²Department of Clinical Physics and Bioengineering, Beatson West of Scotland Cancer Centre, 1053 Great Western Road, Glasgow G12 OYN, Scotland, UK. ${ }^{3}$ Department of Clinical Oncology, Beatson West of Scotland Cancer Centre, 1053 Great Western Road, Glasgow G12 OYN, Scotland, UK. ${ }^{4}$ Clinical Oncology Unit, University of Malaya Cancer Research Institute, Faculty of Medicine, University of Malaya, Kuala Lumpur, Malaysia.

Received: 19 December 2012 Accepted: 24 May 2013

Published: 28 May 2013

\section{References}

1. Staffurth JN: A review of clinical evidence for intensity-modulated radiotherapy. Clin Oncol 2010, 22:643-657.

2. Lam P, Au-Yeung KM, Cheng PW, Wei WI, Yuen AP, Trendell-Smith N, Li JH, Li R: Correlating MRI and histologic tumor thickness in the assessment of oral tongue cancer. Am J Roentgenol 2004, 182:803-808.

3. Tetsumura A, Yoshino N, Amagasa T, Nagumo K, Okada N, Sasaki T: High-resolution magnetic resonance imaging of squamous cell carcinoma of the tongue: an in vitro study. Dentomaxillofac Radiol 2001, 30:14-21.

4. Roland NJ, Paleri V: Head and Neck Cancer: Multidisciplinary Management Guidelines. 4th edition. London: ENT UK; 2011.

5. Chung NN, Ting LL, Hsu WC, Lui LT, Wang PM: Impact of magnetic resonance imaging versus CT on nasopharyngeal carcinoma: primary tumor target delineation for radiotherapy. Head Neck 2004, 26:241-246.

6. Ahmed M, Schmidt M, Sohaib A, Kong C, Burke K, Richardson C, Usher M, Brennan S, Riddell A, Davies M, Newbold K, Harrington KJ, Nutting CM: The value of magnetic resonance imaging in target volume delineation of base of tongue tumours - a study using flexible surface coils. Radiother Oncol 2010, 94:161-167.

7. Newbold K, Partidge M, Cook G, Sohaib SA, Charles-Edwards E, Rhys-Evans $P$, Harrington $K$, Nutting $C$ : Advanced imaging applied to radiotherapy planning in head and neck cancer: a clinical review. Br J Radio/ 2006, 79:554-561.

8. Sefrova J, Odrazka K, Paluska P, Belobradek Z, Brodak M, Dolezel M, Prosvic P, Macingova Z, Vosmik M, Hoffmann P, Louda M, Nejedla A: Magnetic resonance imaging in postprostatectomy radiotherapy planning. Int $J$ Radiat Oncol Biol Phys 2012, 82:911-918.

9. Smith WL, Lewis C, Bauman G, Rodrigues G, D'Souza D, Ash R, Ho D, Venkatesan V, Downey D, Fenster A: Prostate volume contouring: a 3D analysis of segmentation using 3DTRUS, CT, and MR. Int J Radiat Oncol Biol Phys 2007, 67:1238-1247.

10. Khoo VS, Adams EJ, Saran F, Bedford JL, Perks JR, Warrington AP, Brada M: A comparison of clinical target volumes determined by CT and MRI for the radiotherapy planning of base of skull meningiomas. Int J Radiat Oncol Biol Phys 2000, 46:1309-1317.

11. Prabhakara R, Haresh KP, Ganesh T, Joshi RC, Julka PK, Rath GK: Comparison of computed tomography and magnetic resonance based target volume in brain tumors. J Canc Res Ther 2007, 3:121-123.

12. Doran SJ, Charles-Edwards L, Reinsberg SA, Leach MO: A complete distortion correction for MR images: I. Gradient warp correction. Phys Med Biol 2005, 50:1343-1361.

13. Khoo VS, Joon DL: New developments in MRI for target volume delineation in radiotherapy. $\mathrm{Br} J$ Radiol 2006, 79:S2-S15.

14. Tamhane1 AA, Arfanakis K: Motion correction in PROPELLER and Turboprop-MRI. Magn Reson Med 2009, 62:174-182.

15. Fransson A, Andreo P, Pötter R: Aspects of MR image distortions in radiotherapy treatment planning. Strahlenther Onkol 2001, 177:59-73.

16. Schenck JF: The role of magnetic susceptibility in magnetic resonance imaging: MRI magnetic compatibility of the first and second kinds. Med Phys 1996, 23:815-850.

17. Hanvey S, Sadozye AH, McJury M, Glegg M, Foster J: The influence of MRI scan position on image registration accuracy, target delineation and calculated dose in prostatic radiotherapy. Br J Radiol 2012, 85:e1256-e1262.

18. Hanvey S, Glegg M, Foster J: Magnetic resonance imaging for radiotherapy planning of brain cancer patients using immobilization and surface coils. Phys Med Biol 2009, 54:5381-5394. 
19. Kenneth U, Urie MM, Cherlow JM: Results of a multi-institutional benchmark test for cranial CT/MR image registration. Int I Radiat Oncol Biol Phys 2010, 77:1584-1589.

20. Zou KH, Warfield SK, Bharatha A, Tempany CM, Kaus MR, Haker SJ, Wells WM 3rd, Jolesz FA, Kinis R: Statistical validation of image segmentation quality based on a spatial overlap index. Acad Radiol 2004, 11:178-178

21. Grégoire V, Levendag P, Ang KK, Bernier J, Braaksma M, Budach V, Chao C, Coche E, Cooper JS, Cosnard G, Eisbruch A, El-Sayed S, Emami B, Grau C,

Hamoir M, Lee N, Maingon P, Muller K, Reychler H: CT-based delineation of lymph node levels and related CTVs in the node-negative neck: DAHANCA, EORTC, GORTEC, NCIC, RTOG consensus guidelines. Radiother Oncol 2003, 69:227-236.

22. Yoon M, Park SY, Shin D, Lee SB, Pyo HR, Kim DY, Cho KH: A new homogeneity index based on statistical analysis of the dose-volume histogram. J Appl Clin Med Phys 2007, 8:9-17.

23. Prestwich RJD, Sykes J, Carey B, Sen M, Dyker KE, Scarsbrook AF: Improving target definition for head and neck radiotherapy: a place for magnetic resonance imaging and 18-fluoride fluorodeoxyglucose positron emission tomography? Clin Oncol 2012, 24:577-589.

24. Rosenman JG, Miller EP, Tracton G, Cullip TJ: Image registration: an essential part of radiation therapy planning. Int I Radiat Oncol Biol Phys 1998, 40:197-205.

25. Goitein M, Niemierko A: Intensity-modulated therapy and inhomogeneous dose to the tumor: a note of caution. Int I Radiat Oncol Biol Phys 1996, 36:519-522.

doi:10.1186/1748-717X-8-129

Cite this article as: Hanvey et al:: The influence of MRI scan position on patients with oropharyngeal cancer undergoing radical radiotherapy. Radiation Oncology 2013 8:129.

\section{Submit your next manuscript to BioMed Central and take full advantage of:}

- Convenient online submission

- Thorough peer review

- No space constraints or color figure charges

- Immediate publication on acceptance

- Inclusion in PubMed, CAS, Scopus and Google Scholar

- Research which is freely available for redistribution 